\section{THU0177 PROGNOSTIC FACTORS OF REMISSION IN EARLY RHEUMATOID ARTHRITIS (RA). A MULTIPARAMETER PROSPECTIVE STUDY}

${ }^{1} \mathrm{~B}$ Combe, ${ }^{2} \mathrm{P}$ Goupille, ${ }^{3} \mathrm{~A}$ Cantagrel, ${ }^{4} \mathrm{MC}$ Bozonnat, ${ }^{5} \mathrm{~J}$ Sibilia, ${ }^{6} \mathrm{JF}$ Eliaou, ${ }^{7} \mathrm{O}$ Meyer, 1J Sany, ${ }^{4} \mathrm{JP}$ Daures, ${ }^{8} \mathrm{M}$ Dougados. ${ }^{1}$ Rhumatologie; ${ }^{2}$ Rhumatologie, Trousseau, Tours; ${ }^{3}$ Rhumatologie, Rangueil, Toulouse; ${ }^{4} I U R C ;{ }^{5}$ Rhumatologie, Hautepierre, Strasbourg, France; ${ }^{6}$ Immunologie, Lapeyronie, Montpellier; ${ }^{7}$ Rhumatologie, Bichat, Paris; ${ }^{8}$ Rhumatologie, Cochin, Paris

10.1136/annrheumdis-2001.1079

\section{Background}

Objectives To determine prognostic factors of remission in early RA.

Methods A cohort of 191 patients with RA and disease duration shorter than 1 year were prospectively followed-up for 3 years. Numerous baseline clinical, laboratory, genetic and radiographic data (Sharp's method modified by van der Heijde) were obtained. Remission, defined by a Disease Activity Score (DAS) $<1.6$, was used as outcome measure. ${ }^{1}$

Results Forty eight patients (25.1\%) fulfilled the remission criteria at the 3-year follow-up visit. According to Fisher?s exact test, remission was closely related with baseline values of DAS, HAQ score, morning stiffness, tender joint count, C-reactive protein and radiologic scores. No statistically significant correlation was demonstrated for sex, age, extraarticular manifestations, IgM and IgA rheumatoid factors, anti-perinuclear $\mathrm{Ab}$, anti-keratin $\mathrm{Ab}$, anti-HSP 90, anti-calpastatin Ab, antinuclear Ab, YKL-40 and HLA-DRB1* genotypes.

The logistic regression analysis revealed that the independent variables able to predict the 3-year remission were: DAS, HAQ score, morning stiffness and total radiographic score.

Conclusion Pronostic factors of remission in early RA were mainly clinical markers of disease activity and radiological scores.

\section{REFERENCE}

1 Prevoo, et al. Br J Rheumatol. 1996;35:1101

\section{THU0178 CLINICAL SIGNIFICANCE OF RAPID RADIOGRAPHIC PROGRESSION IN RHEUMATOID ARTHRITIS}

${ }^{1} \mathrm{JB}$ Wong, ${ }^{2} \mathrm{CJ}$ Wong, ${ }^{3} \mathrm{JM}$ Hazes, ${ }^{4} \mathrm{PL}$ Van Riel, ${ }^{5} \mathrm{FC}$ Breedveld, ${ }^{6} \mathrm{DM}$ Van der Heidje, ${ }^{2}$ BG Feagan. 'Medicine-Clinical Decision Making, Tufts-New England Medical Center, Boston, USA; ${ }^{2}$ London Clinical Trials Research Group, Robarts Research Institute, London, Canada; ${ }^{3}$ Rheumatology, University Hospital, Rotterdam; ${ }^{4}$ Rheumatology, University Hospital, Nijmegen; ${ }^{5}$ Rheumatology, Leiden University Medical Center, Leiden; ${ }^{6}$ Rheumatology, University Hospital, Maastricht, Netherlands

\subsection{6/annrheumdis-2001.1080}

Background New treatments for rheumatoid arthritis have established radiographic stabilisation of joint disease as an achievable therapeutic goal, but the amount of radiologic progression that is clinically significant remains uncertain. OMERACT has defined significant radiographic progression by the smallest detectable difference (SDD) based on random measurement error calculated from between observer variation in pairwise radiographic scoring and interpretation.

Objectives To examine the clinical significance of SDD-defined radiographic progression.

Methods We combined 2 Dutch cohorts of early rheumatoid arthritis patients from Nijmegen and Leiden. Using an SDD of 15 for the modified Sharp score, 279 patients had year 2 or 3 radiographs (mean age 50.5 year, $76.7 \%$ women, $71.7 \% \mathrm{RF}+$,
60.1\% HLA DR4+) and were divided into 2 groups based on their annual radiographic progression rate: rapid ( $>7.5$ ) vs nonrapid $(<7.5)$ radiographic progressors. Exploratory statistical analyses were performed using the Chi-square and Wilcoxon rank sum tests.

Results In this cohort, 199 of the 279 patients (71\%) were ? rapid progressors? after $2-3$ years of RA. At that time, fast progressors tended to be HLA DR4+ $(p=0.11)$ but had higher ESR, Ritchie articular index, HAQ and Disease Activity Scores (DAS) (all p < 0.02), and were more likely to be older and RF+ (all $\mathrm{p}<0.0001$ ). After $5-6$ years of RA, fast progressors had higher median modified Sharp scores $(102$ vs 8 , p $<0.0001)$, HAQ scores $(0.50$ vs $0.23, \mathrm{p}<0.003)$, and DAS $(2.80$ vs 2.02 , $\mathrm{p}=0.003)$. After 12 years of RA, fast progressors had higher median HAQ (0.88 vs $0.26, \mathrm{p}=0.013)$ and DAS (2.88 vs 2.35 , $\mathrm{p}=0.11$ ).

Conclusion Although SDD-based radiographic progression is defined as a statistically significant worsening beyond random measurement error, such radiologic progression of joint disease appears to identify rheumatoid arthritis patients who are significantly more likely to experience clinically progressive disease. Identifying and targeting the use of new biologic therapies toward this group of radio logically rapidly progressive rheumatoid arthritis patients is likely to yield the most favourable costeffectiveness ratios because in the absence of these new treatments, these patients are at higher risk of developing progressive disability.

\section{THU0179 MAGNETIC RESONANCE IMAGING OF THE HAND IN EARLY RHEUMATOID ARTHRITIS}

${ }^{1}$ I Ertenli, ${ }^{2} \mathrm{D}$ Altınok, 'S Kiraz, ${ }^{2} Y$ Karadeniz, ${ }^{1} \mathrm{MA}$ Oztürk, ${ }^{1} \mathrm{~S}$ Apras, ${ }^{1} \mathrm{M}$ Çalgüneri. ${ }^{1}$ Rheumatology, Hacettepe University School of Medicine, Ankara; ${ }^{2}$ Radiology, Kirrkkale University Medical School, Kirikkale, Turkey

\subsection{6/annrheumdis-2001.1081}

\section{Background}

Objectives This study is planned as a prospective investigation of the accuracy of magnetic resonance imaging for the demonstration of early-stage rheumatoid arthritis findings of both hands, with the goal of differentiating magnetic resonance imaging and plain film findings of the patients having early -stage rheumatoid arthritis.

Methods Ten patients who met American Rheumatism Association criteria for rheumatoid arthritis and symptoms of less than one year duration were accepted for the study. After full clinical assessment, laboratory tests and radiographs of the hands were undertaken, magnetic resonance imaging was performed on a 1.5 tesla imager using an extremity coil using SE T1, TSE T2, fat suppressed T2, flash 2d 3DGRE and postcontrast fat suppressed $\mathrm{T} 1$ weighted sequences.

Results 6 of 10 patients had periarticular osteoporosis on plain radiography. All of the 10 patients had subcondral erosions, 8 patients had effusion in proximal interphalangeal joints, 8 had synovial hypertrophy, 7 had tendonitis, 5 had tendon erosions and tears and 2 had ulnar collateral ligament ruptures.

Conclusion Magnetic resonance imaging not only allows direct visualisation of the inflammatory soft tissue, but also its effect on the bone, cartilage, tendons and ligaments. Magnetic resonance imaging, due to its superior soft-tissue contrast and tomographic changes is more sensitive to than plain radiography in demonstrating the changes due to rheumatoid arthritis. This may contribute to more accurate diagnosis in early-stage rheumatoid 